Nota científica

\title{
Nueva población de la tortuga de poza del suroeste Emys pallida en el Desierto Central de Baja California, México
}

\author{
New population of the Southwestern pond Turtle Emys pallida in the \\ Central Desert of Baja California, Mexico
}

\author{
Jorge H. Valdez-Villavicencio ${ }^{\text {a,* }}$, Anny Peralta-García ${ }^{a}$ y J. Ángel Guillen-González ${ }^{b}$ \\ ${ }^{a}$ Conservación de Fauna del Noroeste, Calle A, \#33, Villas de Real III, 22785, Ensenada, Baja California, México \\ ${ }^{\mathrm{b}}$ Gestión Ambiental y Desarrollo, 16 de Septiembre, Col. Hidalgo, 22880, Ensenada, Baja California, México \\ Recibido el 26 de mayo de 2015; aceptado el 6 de octubre de 2015 \\ Disponible en Internet el 13 de febrero de 2016
}

\begin{abstract}
Resumen
Se registra una nueva población de la tortuga de poza del suroeste Emys pallida en Baja California. Este registro representa una ampliación en la distribución de $95.5 \mathrm{~km}$ al sur del registro previo más sureño. También representa la única población de oasis dentro de la ecorregión del Desierto Central en Baja California.

Derechos Reservados @ 2015 Universidad Nacional Autónoma de México, Instituto de Biología. Este es un artículo de acceso abierto distribuido bajo los términos de la Licencia Creative Commons CC BY-NC-ND 4.0.
\end{abstract}

Palabras clave: Emydidae; Oasis; Ambiente desértico; Relicto

\begin{abstract}
We report a new population of the southwestern pond turtle Emys pallida in Baja California. This record represents a range extension of $95.5 \mathrm{~km}$ south from the nearest reported location. Also represents the only oasis population within the Central Desert ecoregion in Baja California.

All Rights Reserved @ 2015 Universidad Nacional Autónoma de México, Instituto de Biología. This is an open access item distributed under the Creative Commons CC License BY-NC-ND 4.0.
\end{abstract}

Keywords: Emydidae; Oasis; Desert environment; Relict

La tortuga de poza del suroeste Emys pallida (=Actinemys marmorata pallida) es una de las 3 especies nativas de la familia Emydidae que habitan en el oeste de Norteamérica, y la única tortuga de agua dulce nativa en el estado de Baja California (Spinks, Thomson y Shaffer, 2014). Es una tortuga pequeña cuyo caparazón alcanza los 200 mm de longitud (Grismer, 2002; fig. 1), y se encuentra asociada a hábitats ribereños en la región mediterránea de Baja California. Se distribuye desde la bahía de San Francisco en California hasta el arroyo El Rosario en Baja California (Grismer, 2002; Stebbins, 2003). Esta especie

\footnotetext{
* Autor para correspondencia.

Correo electrónico: j_h_valdez@yahoo.com.mx

(J.H. Valdez-Villavicencio).

La revisión por pares es responsabilidad de la Universidad Nacional Autónoma de México.
}

ha sufrido declives drásticos en las poblaciones de California, Estados Unidos de América, y posiblemente también en Baja California (Bury y Germano, 2008; Pramuk et al., 2013); sin embargo, se desconoce su estado actual.

Como parte de un estudio preliminar sobre la distribución actual de E. pallida en Baja California se consultó la colección herpetológica de la Facultad de Ciencias de la Universidad Autónoma de Baja California, donde se encontró el ejemplar recolectado por Clementina Gerardo e Israel Hernández, el 11 de abril de 2009, en el oasis San José, Rancho San José, en el ejido San José de las Palomas, Baja California. Para verificar la ampliación en la distribución de la especie se visitó la localidad el 29 y el 30 de julio de 2014. La localidad se ubica a $40 \mathrm{~km}$ al sur de Cataviña $\left(29.36462^{\circ} \mathrm{N}, 114.74900^{\circ} \mathrm{O}\right.$, elevación $107 \mathrm{~m}$ ). El oasis consta de un tramo del arroyo San José de aproximadamente $5 \mathrm{~km}$ de longitud, en el cual se mantiene flujo 


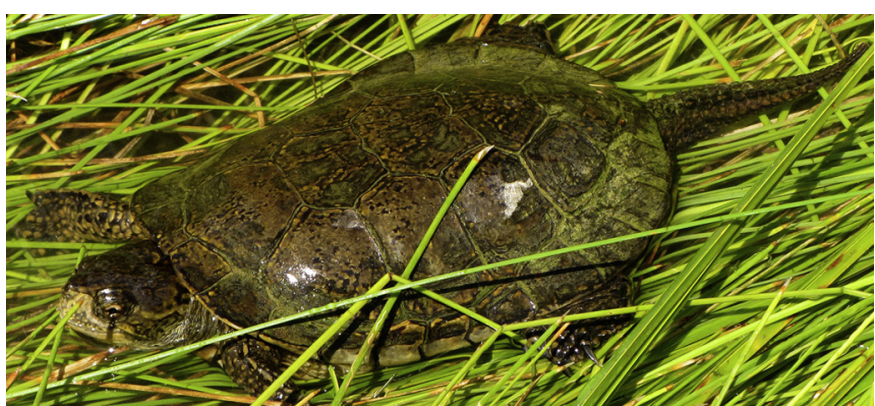

Figura 1. Ejemplar de Emys pallida del oasis San José, Baja California, México.

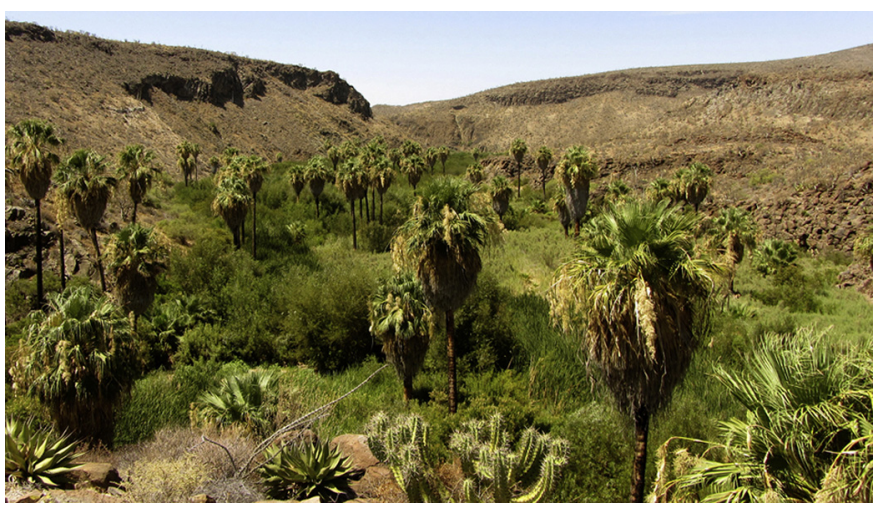

Figura 2. Vista del oasis San José en la ecorregión del Desierto Central de Baja California.

de agua superficial con pozas de manera permanente. El hábitat presenta una densa vegetación riparia en la que predominan la palma abanico (Washingtonia robusta), el sauce (Salix lasiolepis), la hierba del manso (Anemopsis californica), el guatamote (Baccharis salicifolia) y el junco (Juncus acutus) (fig. 2).

Se colocaron 2 trampas tipo nasa de $45 \mathrm{~cm}$ de diámetro y $1 \mathrm{~m}$ de longitud separadas $15 \mathrm{~m}$ entre sí, cebadas con sardinas (Bury et al., 2012), en una poza de $500 \mathrm{~m}^{2}$ aproximadamente, bordeada por juncos (J. acutus), tule (Schoenoplectus californicus) y tule petatero (Typha domingensis). Las trampas se colocaron a las 08:30 h y se revisaron a las 07:00 h del día siguiente. Para cada captura se tomaron medidas del caparazón-largo, ancho y alto-, largo del plastrón, peso y sexo (U. S. Geological Survey, 2006), y posteriormente se liberaron los individuos en el lugar de captura. También se midieron los parámetros físico-químicos del agua con el propósito de realizar futuras comparaciones de la calidad del agua. Los parámetros medidos fueron: temperatura del agua, $\mathrm{pH}$, conductividad, sólidos disueltos totales y salinidad, utilizando un medidor multiparámetros -modelo 35, Eutech/Oakton

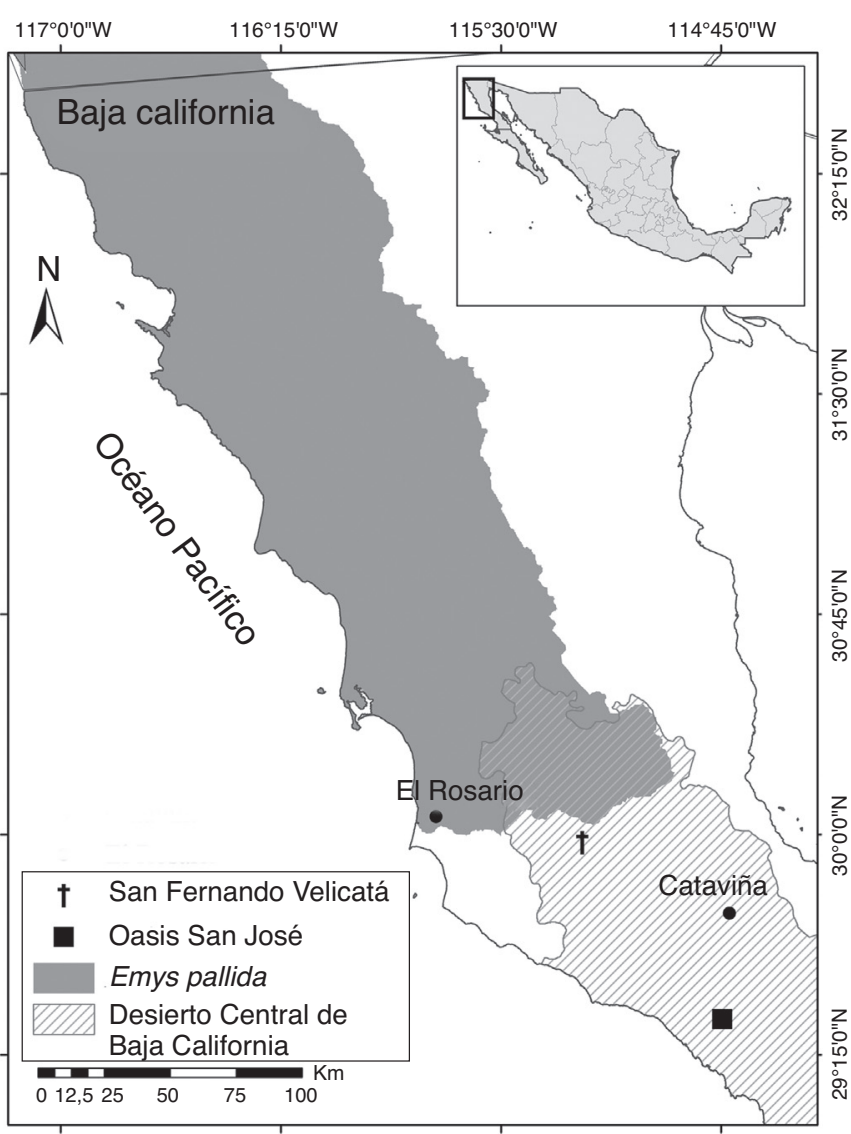

Figura 3. Distribución de Emys pallida en Baja California. El cuadrado negro indica la nueva población en el oasis San José, y la cruz, la población extinta en la Misión San Fernando Velicatá. Modificada de Grismer, 2002.

Instruments, Vernon Hills, IL, Estados Unidos de América-, además de oxígeno disuelto con un oxímetro -modelo YSI, YSI Inc., Yellow Spring, OH, Estados Unidos de América-.

Se capturaron 10 tortugas adultas ( 2 hembras y 8 machos; tabla 1) y se observaron otros 7 individuos en un trayecto de $1.5 \mathrm{~km}$ arroyo arriba desde la poza. Los parámetros físicoquímicos del agua en el sitio de captura fueron: temperatura del agua $=21.5^{\circ} \mathrm{C}, \mathrm{pH}=6.54$, conductividad $=3.25 \mathrm{mS} / \mathrm{cm}$, sólidos disueltos totales $=2.33 \mathrm{ppt}$, salinidad $=1.6 \mathrm{ppt}$ y oxígeno disuelto $=126.1 \%$ y $10.9 \mathrm{mg} / \mathrm{L}$. Esta población de E. pallida representa una ampliación en su distribución geográfica de $95.5 \mathrm{~km}$ al sur del registro más cercano en el arroyo El Rosario (Grismer, 2002; fig. 3). Asimismo, aunque Roberts (1982) registró la primera población de oasis en un ambiente desértico, esta se considera extinta en la actualidad (Grismer, 2002), por

Tabla 1

Medidas de los individuos capturados de Emys pallida en el oasis San José, Baja California.

\begin{tabular}{|c|c|c|c|c|c|c|}
\hline Sexo & $\mathrm{n}$ & \multicolumn{3}{|c|}{ Caparazón (mm) } & Plastrón (mm) & Peso (g) \\
\hline Hembras & 2 & $\begin{array}{l}126.2 \\
(124.7-127.6)\end{array}$ & $\begin{array}{l}43.5 \\
(43.3-43.7)\end{array}$ & $\begin{array}{l}93.7 \\
(92.5-94.8)\end{array}$ & $107^{\mathrm{a}}$ & $\begin{array}{l}273.5 \\
(265-282)\end{array}$ \\
\hline Machos & 8 & $\begin{array}{l}120.1 \\
(103.8-128.9)\end{array}$ & $\begin{array}{l}39 \\
(33.8-45.1)\end{array}$ & $\begin{array}{l}87.4 \\
(78.1-93.9)\end{array}$ & $\begin{array}{l}100.3 \\
(90.6-108.6)\end{array}$ & $\begin{array}{l}228.4 \\
(152-282)\end{array}$ \\
\hline
\end{tabular}

\footnotetext{
a Ambas hembras presentaron la misma medida.
} 
lo que la población del oasis San José representa la única población actual de oasis en la ecorregión de Desierto Central de Baja California (González-Abraham, Garcillán, Ezcurra y Grupo de Trabajo Ecorregiones, 2010).

Grismer (2002) considera que esta especie se originó en el noroeste de Norteamérica y se dispersó a Baja California con la aparición de un corredor mésico por los rangos montañosos durante el Pleistoceno, limitando su distribución sureña por la formación del desierto de El Vizcaíno. El oasis San José pudo haberle proporcionado refugio ante la desertificación de la península hace 8,000 a 10,000 años, quedando aislada, como ha ocurrido en otras especies de afinidad mésica en otros oasis de la península (Grismer y McGuire, 1993). Por otro lado, no se puede descartar la posibilidad de que la especie haya sido translocada, tal como se ha registrado para la población más sureña en el arroyo El Rosario (Welsh, 1988). Sin embargo, los dueños del predio mencionan que las tortugas han estado ahí desde que se estableció el rancho en la localidad, en 1949 (C. Gerardo com. pers.). Actualmente el rancho se encuentra deshabitado y sus dueños solo lo visitan esporádicamente debido a su lejanía y a la inaccesibilidad del lugar. Esto ha permitido que el oasis permanezca prístino y en buen estado de conservación. Además, el predio donde se encuentra el oasis forma parte del programa de pago por derechos ambientales de la Semarnat-Conafor para conservación de biodiversidad; asimismo, se localiza dentro del Área de Protección de Flora y Fauna Valle de los Cirios (Conanp, 2013).

Agradecemos a Clementina Gerardo, del Rancho San José, por el acceso al sitio de estudio, así como a los revisores anónimos por sus comentarios y sugerencias en la versión previa del manuscrito.

\section{Referencias}

Bury, R. B., Ashton, A. T., Germano, D. J., Karraker, N. E., Reese, D. A. y Schelick, K. E. (2012). Sampling of turtles: trapping and snorkeling. En R. B. Bury, H. H. Welsh, D. J. Germano y A. T. Ashton (Eds.), Western pond turtle:
Biology, sampling techniques, inventory and monitoring, conservation, and management (7). Olympia, Washington: Northwest Fauna.

Bury, R. B. y Germano, D. J. (2008). Actinemys marmorata (Baird and Girard 1852)-Western pond turtle, Pacific pond turtle. En A. G. Rhodin, P. C. H. Pritchard, P. P. van Dijk, R. A. Saumure, K. A. Buhlmann y J. B. Iverson (Eds.), Conservation biology of freshwater turtles and tortoises: A compilation project of the IUCN/SSC Tortoise and Freshwater Turtle Specialist Group (5) (pp. 001.1-001.9). Lunenburg, Massachusetts: Chelonian Research Monographs.

Conanp. (2013). Programa de manejo del área de protección de flora y fauna Valle de los Cirios. México, D. F.: Secretaría de Medio Ambiente y Recursos Naturales y Comisión Nacional de Áreas Naturales Protegidas.

González-Abraham, C. E., Garcillán, P. P., Ezcurra, E., \& Grupo de Trabajo Ecorregiones. (2010). Ecorregiones de la península de Baja California: una síntesis. Boletín de la Sociedad Botánica de México, 87, 69-82.

Grismer, L. L. (2002). Amphibians and reptiles of Baja California, including its Pacific islands and the islands in the Sea of Cortés. Berkeley, California: University of California Press.

Grismer, L. L. y McGuire, J. A. (1993). The oases of central Baja California, Mexico. Part I. A preliminary account of the relict mesophilic herpetofauna and the status of the oases. Bulletin of the Southern California Academy of Sciences, 92, 2-24.

Pramuk, J., Koontz, F., Tirhi, M., Zeigler, S., Schwartz, K. y Miller, P. (2013). The Western pond turtle in Washington: a population and habitat viability assessment. Apple Valley, Minnesota: IUCN/SSC Conservation Breeding Specialist Group.

Roberts, N. (1982). A preliminary report on the status of Chelydridae, Trionychidae, and Testudinidae in the region of Baja California, Mexico. Proceedings of the Desert Tortoise Council, 154-161.

Spinks, P. Q., Thomson, R. C. y Shaffer, H. B. (2014). The advantages of going large: genome-wide SNPs clarify the complex population history and systematics of the threatened western pond turtle. Molecular Ecology, 23, 2228-2241.

Stebbins, R. C. (2003). A field guide to western reptiles and amphibians (3rd ed.). Boston: Houghton Mifflin Company.

U. S. Geological Survey (USGS). (2006). USGS Western pond turtle (Emys marmorata) trapping survey protocol for the southcoast ecoregion. San Diego, California: U. S. Geological Survey Protocol.

Welsh, H. H. (1988). An ecogeographic analysis of the herpetofauna of the Sierra San Pedro Martir region, Baja California, with a contribution to the biogeography of the Baja California herpetofauna. Proceedings of the California Academy of Sciences, 46, 1-72. 